\title{
Energy and Bandwidth Constrained QoS Enabled Routing for MANETs
}

\author{
N.Sumathi ${ }^{1}$ and Dr.C.P.Sumathi ${ }^{2}$ \\ ${ }^{1}$ Department of Computer Applications, S.N.R.Sons College, Coimbatore \\ sumi_karivaradaneyahoo.co.in \\ ${ }^{2}$ Department of Computer Science, SDNB Vaishnav College for Women, Chennai
}

\begin{abstract}
Mobile adhoc networks are rapid deployable self organizing networks. Their key characteristics are dynamic topology, high node mobility, low channel bandwidth and limited battery power. Hence, it is necessary to minimize bandwidth and energy consumption. To transmit packets, available bandwidth is known along the route from sender to receiver. Thus, bandwidth estimation is the main metric to support Quality of Service (QoS). This work focuses on improving the accuracy of available bandwidth and incorporating a QoS-aware scheme into the route discovery procedure. It is also important to limit the energy consumed by nodes. Probability based overhearing method is proposed to reduce energy spent on overhearing nodes. This experiment is implemented in NS2 simulator and the performance of the network is analyzed in terms of QoS parameters.
\end{abstract}

\section{KEYWORDS}

Backoff, Malicious Attacks, MANETs, Overhearing, QoS

\section{INTRODUCTION}

Mobile adhoc network (MANET) consists of collection of wireless nodes that have a significantly lower capacity than wired networks [1]. MANETs are typically used in military applications, law enforcement, disaster recovery, emergency search and rescue operations. Due to its constantly changing environment and bandwidth constraint, supporting Quality of Service (QoS) is a challenging task. QoS routing is the process of providing end-to-end and loop free path to ensure the necessary QoS parameters such as delay, bandwidth, probability of packet loss, delay variance (jitter), etc. Energy conservation is another QoS attribute which is taken into consideration.

One of the key research problems in MANETs is routing. The routing protocols establish an efficient route between two nodes so that messages can be delivered in an effective way. Numerous protocols have been developed for MANETs [2]. Such protocols must deal with the typical limitations of these networks, such as low bandwidth, high power consumption, and high error rates. AODV (Adhoc Ondemand Distance Vector) is a reactive routing protocol for ad hoc and mobile networks [3][4] that maintains routes only between nodes which need to communicate. The routing messages do not contain information about the whole route paths, but only about the source and the destination. Hence, the size of the routing messages is reduced. It uses destination sequence numbers to specify how fresh a route is, which is used to grant loop freedom. 
International Journal of Computer Networks \& Communications (IJCNC) Vol.5, No.2, March 2013

\section{RELATED WORK}

In mobile ad hoc networks, few authors suggested solutions for bandwidth estimation. QoSAODV [5] estimates available bandwidth per node. To estimate the available bandwidth, the authors calculate a metric called BWER (Bandwidth Efficiency Ratio) which is the ratio between the numbers of transmitted and received packets. In [6], bandwidth estimation is enhanced by considering collisions and backoff. In this, value of the available bandwidth on a link depends on both channel utilization ratios and the idle period synchronization. Also, the collision probability is estimated and integrated to the available bandwidth estimation. QoS-aware routing protocol [1] incorporates unused bandwidth estimation and an admission control scheme. But there is no measure to predict a route break.

In the protocol AAC (Adaptive Admission Control), each node estimates its local used bandwidth by adding the size of sent and sensed packets over a fixed time period [7]. It solves intra-flow contention problem by estimating the contention count of nodes along a QoS path. In [8], the authors have considered idle times of both the sender and the receiver to achieve more accuracy. Unfortunately, they have not considered the backoff periods in the estimation technique. Also, more accurate solutions are required to overcome hidden terminal problem. In [9], they have proposed a cross-layer framework to support QoS multicasting and estimate available bandwidth using the passive listening method. Passive Listening method is an efficient way to estimate available bandwidth with no extra control overhead. In [5], to improve the accuracy of available bandwidth estimation, they presented a protocol called ABE-AODV (Available Bandwidth Estimation). The main components of this protocol are estimating node's emission capabilities, link's available bandwidth, idle time synchronization between source and destination, evaluating collision probability and backoff mechanism. Then they have estimated the available bandwidth by considering the above components into account. The authors have not considered the overhead due to backoff mechanism.

Since the channel is shared by all the nodes, there is a chance for collision. To reduce collision, backoff algorithm is proposed. In MILD (Multiple Increase and Linear Decrease) [10], when collision occurs, CW (Contention Window) size is multiplied by 1.5 and decreased by 1 for a successful transmission. MILD performs well when the network load is heavy. In BEB (Binary Exponential Backoff) [11], nodes use the same CW value regardless of number of nodes. So lot of collisions occur and throughput is reduced. At the beginning of each slot a node transmits if its backoff timer has expired. Otherwise depending on the channel state (idle or busy), the node will count down the backoff counter by 1 or will be frozen at a value. Such an algorithm is embedded in IEEE 802.11 DCF. It has the following drawbacks. First, CW is doubled upon failure regardless of the type of failure and second is after a successful transmission of packet, $\mathrm{CW}$ size is reset to $\mathrm{CW}_{\min }$, thus forgetting its knowledge of the current congestion level in the network [12]. Exponential Increase Exponential Decrease (EIED) algorithm [13] and LMILD scheme (Linear/Multiplicative Increase Linear Decrease) [14] out-perform BEB and MILD algorithms for a wide range of network sizes.

In DIDD [15] backoff algorithm (Double Increment Double Decrement) CW decreases smoothly after a successful packet transmission. It achieves better performance than BEB. Log based backoff algorithm is introduced in [16] to improve the throughput performance. Pipelining concept is discussed in [17] for scheduling packet transmissions. To reduce the channel idle time and overhead associated with collision, log based pipelined backoff algorithm is proposed.

Wireless adhoc networks use a wide range of energy conserving techniques. In DPSM [18] (Dynamic Power Saving Mechanism) scheme, the ATIM (Adhoc Traffic Indication Map) window size is adjusted dynamically based on current network conditions. 
A NPSM [19] (New Power Saving Mechanism) introduces some parameters indicating amount of data in each station. In (ODPM) (On Demand Power Management) [20], soft state timers are set or refreshed on-demand based on control messages and data transmission. Nodes that are not involved in data transmission may enter into sleep state to save energy. Energy is saved by integrating routing and MAC layer functionality. The protocol discussed in [21] extends doze time and reduces contention, retransmission and improves channel utilization. It also provides quality of service support. In [22], number of AM (Active Mode) nodes is reduced based on backbone probability. TITAN (Traffic-Informed Topology-Adaptive Network) improves ODPM in which PS nodes sleep for longer duration and saves energy [23].

Rcast [24] implements randomized overhearing but not randomized rebroadcast. Dorsey and Siewiorek [25] discussed a fast wakeup mechanism for route discovery to reduce latency. In Randomcast algorithm [26], sender can specify the desired level of overhearing in order to save energy and reduce redundant rebroadcasts to improve the performance. It is integrated with DSR (Dynamic Source Routing) routing protocol. In DSR, route caches often contain stale route information. It broadcasts more control packets which waste channel capacity and energy. Another cause of excessive energy consumption is redundant rebroadcasting. Redundant rebroadcasts increases network traffic as well as wastes energy resource for transmitting and receiving the broadcasts. To overcome these limitations, AOMDV (Adhoc On demand Multipath Distance Vector) routing protocol is proposed for energy efficient method.

\section{ENERGY EFFICIENT BANDWIDTH CONSTRAINED TECHNIQUE}

Bandwidth estimation is a main function needed to provide QoS in MANETs. Since each host has inaccurate knowledge of the network status and dynamic links, it is difficult to estimate the available bandwidth between nodes. Hence, an effective bandwidth estimation scheme is needed.

\subsection{Available Bandwidth Measurement Algorithm (ABM)}

Step1: Evaluate the capacity of a node and estimate the available bandwidth.

Available bandwidth $=$ Channel Capacity - Utilized Bandwidth

Here Utilized Bandwidth $=\mathrm{N} * \mathrm{~S} * 8 / \mathrm{T}$

where N- No. of packets, S- Size of packet and T- Time duration

Step 2: Estimate the link's available bandwidth. It depends on channel utilization ratio and idle period synchronization. Let it be $\mathrm{E}(\mathrm{b}(\mathrm{s}, \mathrm{r}))$. It is calculated based on the probability that the medium is free simultaneously at the sender and the receiver side.

Step 3: Estimate collision probability $\mathrm{Pm}=\mathrm{f}(\mathrm{m}) \cdot \mathrm{P}_{\text {hello }}$

where $f(m)$ - Lagrange interpolated polynomial function, $P_{\text {hello }}$ - Collision probability estimated based on hello packets.

$\left(\mathrm{P}_{\text {hello }}=(\right.$ Expected - Recd no. of Hello pkts $) /$ Expected no. of Hello packets $)$

Step 4: Collision leads to retransmission of same frames. When collision occurs, log based pipelined backoff algorithm is executed. Backoff algorithm is used to reduce collisions when more than one node tries to access the common channel. This is an additional overhead which affects the available bandwidth.

Bandwidth loss due to this additional overhead $\mathrm{K}$ is evaluated as

$$
K=\frac{\text { DIFS }+\overline{\text { backoff }}}{T(m)}
$$


where DIFS- DCF Inter Frame Spacing, T(m)- time between two consecutive frames and $\overline{b a c k o f f}$ - the average number of slots decremented for a frame.

The above facts are considered and combined to estimate the final available bandwidth.

$$
E_{\text {final }}(b(s, r))=(1-K) \cdot(1-P) \cdot E(b(s, r)
$$

where $E_{\text {final }}(b(s, r))$ is the available bandwidth on link by monitoring node and link capacities, $\mathrm{P}$ is collision probability and $\mathrm{K}$ is bandwidth loss due to backoff scheme.

Step 5: Finally this estimated available bandwidth is stored in neighbor nodes with the help of hello messages.

Step 6: Malicious nodes consume bandwidth and increases packet loss. These attackers are identified and blocked using a threshold value set for the node.

Step 7: Routing protocol called enhanced link disjoint AOMDV (Adhoc On demand Multipath Distance Vector) finds the route based on this available bandwidth.

The existing algorithm employs BEB (Binary Exponential Backoff) to reduce collision. The main problem with BEB is that the node with a long backoff value moves outside the transmission range before it accesses the channel. Also, proportion of bandwidth is wasted due to collision and channel idle time. BEB follows serial transmission. Channel idle time and contention overhead is more because of this serial transmission. Nodes go through a channel contention and packet transmission stages sequentially. Channel contention stage consumes channel bandwidth. Thus time spent on channel contention is reduced when probability of collision is less. But it is difficult to achieve because channel contention cannot be started until the current transmission finishes. Also access to a slot is not uniform. Only the winners repeatedly get the chance to access the channel. This leads to channel capture effect. In a heavily contended network, the collision probability increases which degrades the performance. To minimize these drawbacks, log based pipelining technique is applied to backoff procedure to reduce the collision overhead and improve the available bandwidth.

Pipelining concept is applied to channel contention procedure of MAC (Medium Access Control) protocol. When two nodes are sharing the channel, the remaining nodes start the channel contention procedure in parallel for the next packet transmission. Pipelined backoff hides channel idle time and reduces collision probability. It is also used to control number of contending nodes. Log based backoff algorithm uses logarithm of current backoff counter to calculate next backoff. In the existing approach, $\mathrm{CW}$ size is doubled on collision and backoff counter depends on this new CW. This increases the chance of losing channel access by a node. When a log value is applied to current backoff counter, the difference between two backoff counters is small. So the waiting time of colliding nodes get reduced which improves the throughput performance. Winning node reduces its contention window size by half. Due to this, channel capture effect is reduced. Stage1 reduces both channel idle time and collision overhead. Stage 2 transmits packets and consumes channel bandwidth. Whenever collision occurs or a node looses channel in stage2, there is no need to double the contention window. This approach reduces the number of nodes in stage2. Bandwidth loss due to this backoff is estimated and final available bandwidth is calculated. Final bandwidth is stored in nodes with the help of Hello messages.

\subsection{Energy efficient method}

Energy saving mechanism is important for the efficient operation of the battery powered networks. All the neighboring nodes overhear when a node is transmitting a packet. Hence it is 
necessary to limit the number of overhearing nodes based on probability. Probability value depends on the number of neighbors. The proposed algorithm controls the number of overhearing nodes. It saves energy consumption without affecting quality of route information. When a node is ready to transmit a frame, check its overhearing level (OL) for broadcast and unicast transmission. Three possibilities such as probability overhearing, no overhearing, and unconditional overhearing are considered while finding the routes. Probability overhearing is defined as that few nodes that satisfies a probability based condition can overhear. No overhearing is one in which only a very minimum number of nodes (sender, receiver and intermediate nodes) can overhear and the others would go to low-power sleep state. Unconditional overhearing is one in which almost all one hop neighbor nodes in a network can overhear. Sender is able to specify the level of overhearing. Sender may choose either no or unconditional or probability overhearing which is specified in ATIM frame control. Unconditional overhearing or probability overhearing is set based on the types of messages that are exchanged. a) Probability overhearing is applied for RREP (Route REPly) and DATA packet. b) RERR (Route ERRor) messages will be assigned unconditional overhearing. The reason is that the link failure should be informed to all the nodes, so that the nodes will not use it for the next time until the path gets ready. c) RREQ (Route REQuest) is a broadcast message and based the probability $\left(\mathrm{P}_{\mathrm{o}}\right)$ values, probability overhearing is set. Each node receives ATIM and ATIM-ACK during an ATIM window and depending on its subtype, node is either in awake or sleep state.

Step $1:$ Check if Destination Address $=$ Broadcast $/$ Unicast

Step 2 : If it is Broadcast, check for whether it is the destination. If so, receive packet.

Step 3 : If it is Unicast, check for the subtype values and decide the level of overhearing.

Step 4 : If the subtype is for conditional overhearing then compare the probability values with the threshold and decide the level of overhearing.

Step 5: Rebroadcasting probability and overhearing probability can be identified

Step 6:Repeat the process 2 to 5.

Probability based overhearing method controls the level of overhearing and forwarding of rebroadcast messages. Node is awakened if unconditional overhearing or probability overhearing is set or if it is a destination node. Each node maintains overhearing probability $\mathrm{P}_{\mathrm{o}}$ and rebroadcast probability $\mathrm{P}_{\mathrm{r}}$.

$$
\begin{aligned}
& \mathrm{P}_{\mathrm{o}}=1 / \mathrm{n} \\
& \mathrm{P}_{\mathrm{r}}=\mathrm{cn} / \mathrm{N}^{2}
\end{aligned}
$$

where $\mathrm{c}$ is a constant, $\mathrm{n}$ - No. of neighbors and $\mathrm{N}$ - Average no of. neighbor's neighbors. Then the energy consumed $\left(\mathrm{E}_{\mathrm{c}}\right)$ by the nodes is calculated as

$\mathrm{E}_{\mathrm{c}}=\Sigma\left(\mathrm{I}_{\mathrm{e}}-\mathrm{R}_{\mathrm{e}}\right) /$ no. of pkts transmitted

where $I_{e}$ is Initial energy and $R_{e}$ is residual energy. If a node's subtype is 1101 , it generates a random number between 0 and 1 and compares it with $P_{0}$. If it is greater than $P_{o}$, node decides to overhear. If it is greater than $\mathrm{P}_{\mathrm{r}}$, node decides to rebroadcast. $\mathrm{P}_{\mathrm{o}}$ and $\mathrm{P}_{\mathrm{r}}$ are decided based on number of neighbors. When the number of neighbors is more, redundancy is more.

The main contribution of this part of the work is to limit the number of overhearing nodes based on probability. It reduces energy consumption without affecting quality of route information. This probability based overhearing is incorporated into log based pipelined ABM and integrated with routing protocol. 
This modified algorithm is also integrated into a routing protocol called enhanced link disjoint multipath AODV (AOMDV) to find the routes from given source to destination based on available bandwidth. When a link failure occurs, the node upstream of the link detects the failure, invalidates its routing table entry for that destination and unicasts an RERR message towards the source. Once the source node receives the RERR, it switches its primary path to the next best alternate link-disjoint path. It is designed mainly for highly dynamic ad hoc networks when route breaks and link failures occur frequently. This method reduces routing overhead and improves the performance of the network.

\section{Performance evaluation and analysis of Results}

This work focuses on improving the accuracy of available bandwidth and incorporating a QoSaware scheme into the route discovery procedure. It is also important to limit the energy consumed by nodes. Probability based overhearing method is proposed to reduce energy spent on overhearing nodes. The performance of energy efficient method is evaluated in terms of QoS characteristics as metrics and simulated using NS2 version ns-allinone-2.34 [27].

Performance analysis of energy efficient pipelined ABM is carried out by setting the simulation parameters as per physical layer standard of 802.11. To achieve optimum result, system parameters must be selected according to traffic condition. The simulation time is $200 \mathrm{~s}$ with a grid size of $1000 \times 1000 \mathrm{~m}$. Random way point mobility model is used with moving speed of 20 $\mathrm{m} / \mathrm{s}$ for CBR (Constant Bit Rate) traffic. Carrier sense range is $550 \mathrm{~m}$ and transmission range is $250 \mathrm{~m}$. Packet size is 512 bytes and channel capacity is 2 Mbps.

The parameters considered to evaluate the performance of QoS aware routing protocol are throughput, average end-end delay, Packet Delivery Ratio (PDR), energy consumption, bandwidth consumption and routing overhead. The graphs from Figure 1 to 6 illustrate the performance metrics for a number of nodes. The results are compared with AODV and the proposed methods. Figure 1 shows the throughput performance of pipelined ABM and energy efficient method. There is moderate improvement in throughput which is almost twice than the existing algorithm. The ability to deliver a high percentage of packets to a destination increases the overall utility of the system. Energy efficient method delivers more number of packets successfully under high load.

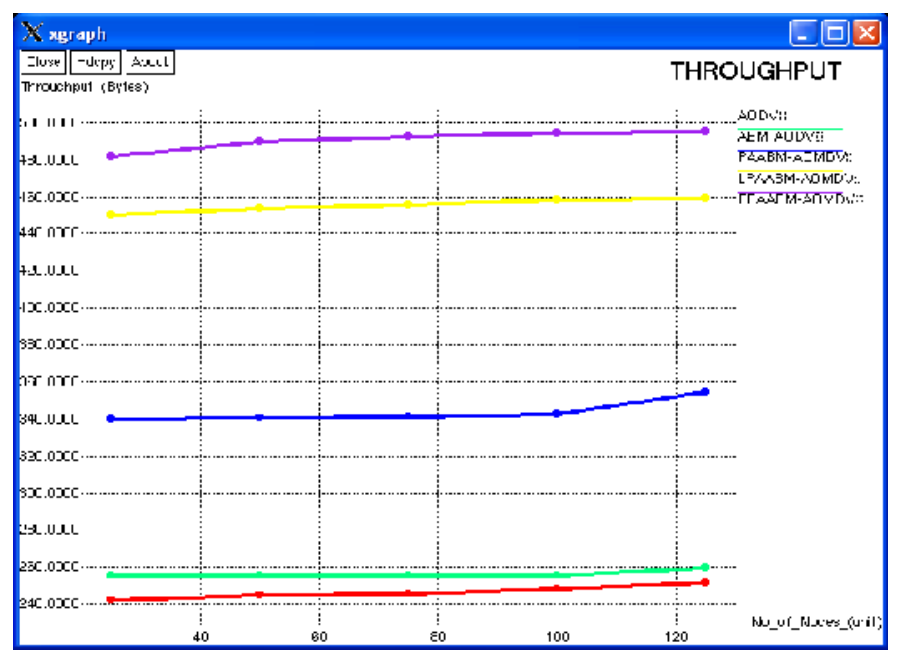

Figure 1. Throughput 
PDR is high because most of the nodes are participated in packet transmission as shown in figure 2. It is observed that proposed method maintains a significantly high PDR than the existing one. This is because the most active path is selected, which has less probability to fail. And in turn increases the PDR by $8 \%$. Average end-end delay is calculated based on the average time required to transmit packets from the source to destination. Figure 3 shows delay caused by energy efficient method varies up to $0.8 \mathrm{~ms}$ whereas in pipelined ABM delay is more.

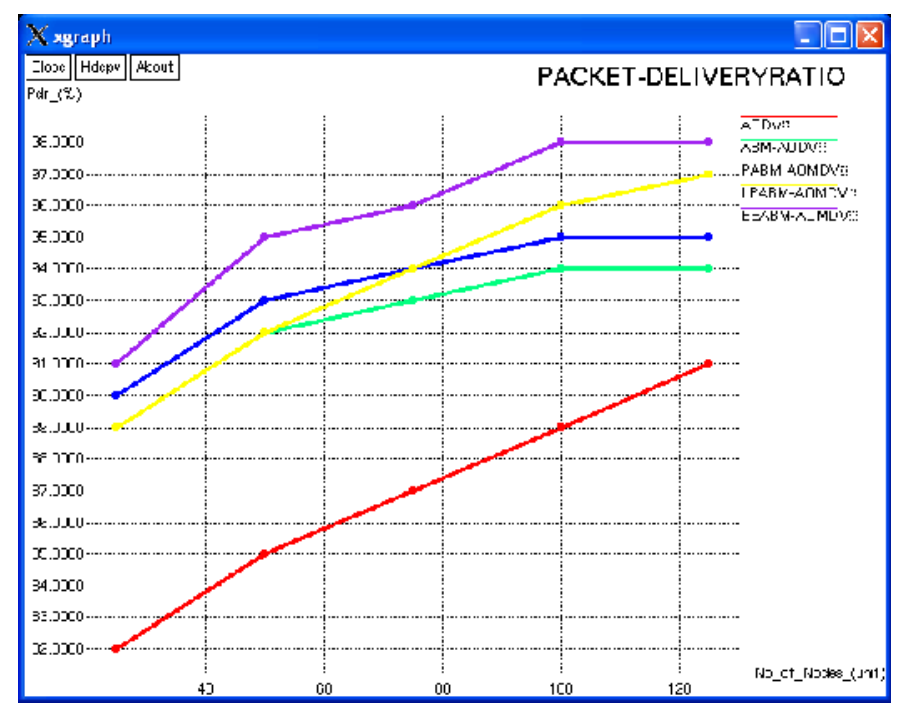

Figure 2. Packet Delivery Ratio

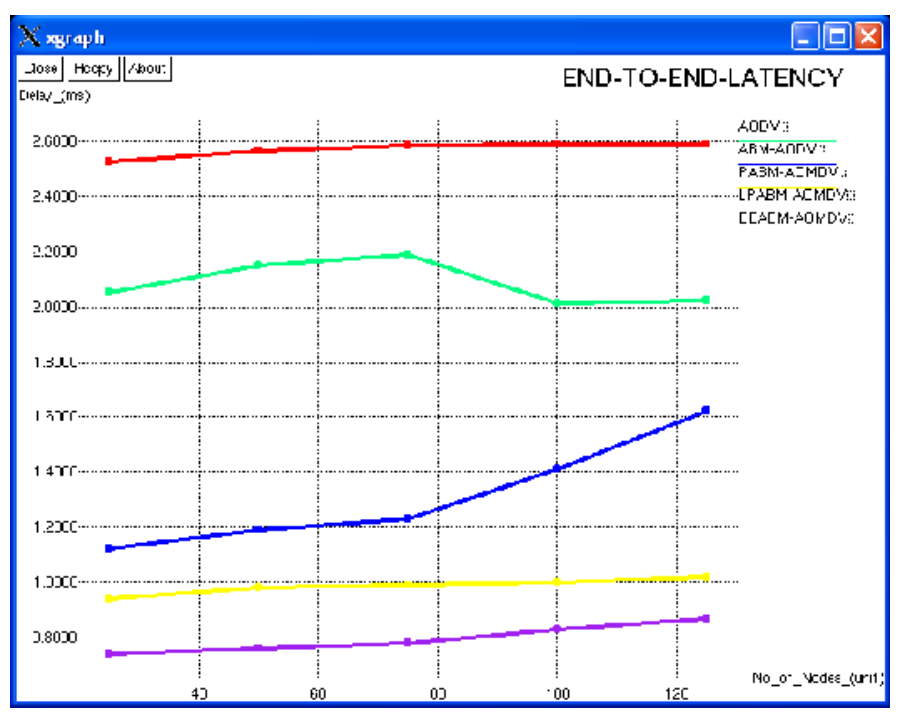

Figure 3. End - End Delay

Figure 4 shows bandwidth consumption. Energy efficient method saves bandwidth compared to other methods. Bandwidth consumption is reduced considerably because of pipelined concept applied to backoff scheme. Probability based overhearing method outperforms other algorithms with respect to energy consumption. Energy efficient pipelined ABM shows less energy consumption than pipelined $\mathrm{ABM}$ as in figure 5. By reducing number of overhearing nodes, 
energy consumption is also reduced. Energy efficient scheme achieves better energy performance under high traffic condition. Performance gap is not dramatic under low traffic condition.

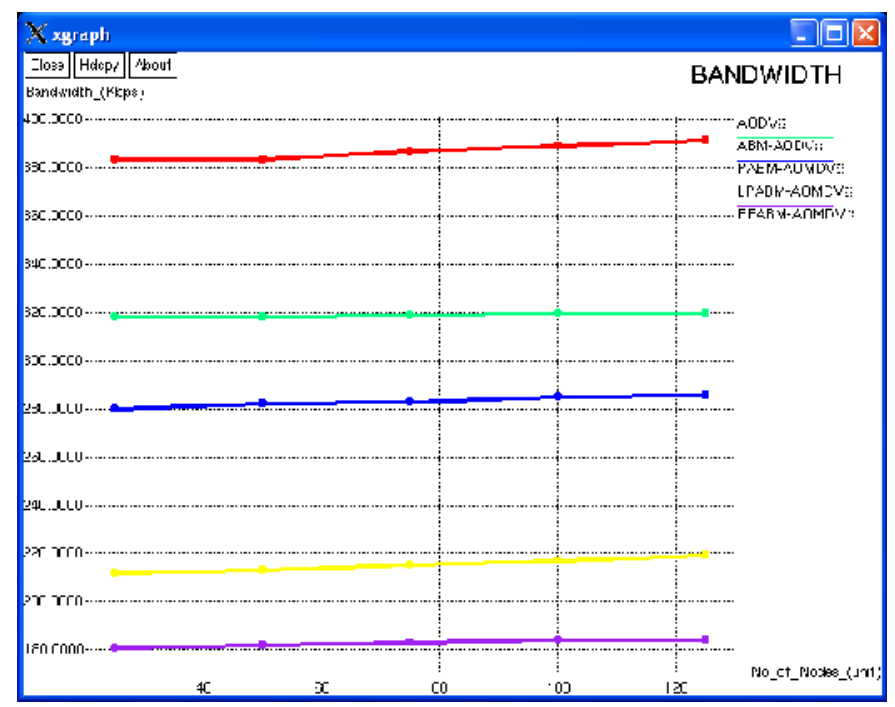

Figure 4. Bandwidth Consumption

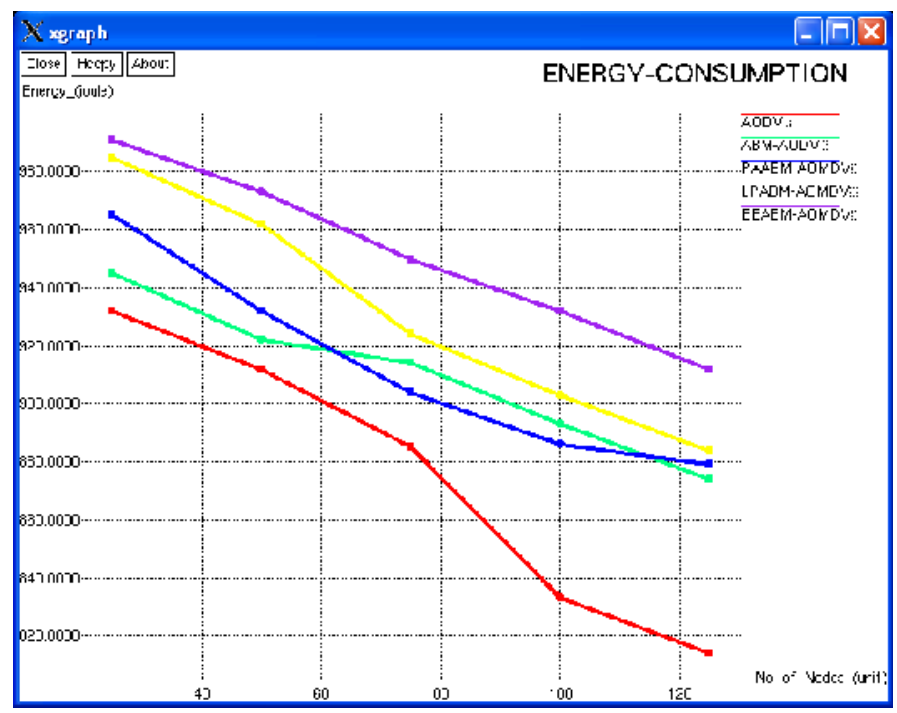

Figure 5. Energy Consumption

Pipelined scheme consumes more energy since nodes keep awake during the entire period of simulation time. In energy efficient scheme, nodes in the range of active communication overhear probabilistically. This is due to the variation of the transmit power between two nodes and also reduction in the number of overhearing nodes. This proposed approach with energy management still reduces the energy consumption. There is not much change in PDR and throughput before and after energy management. Routing overhead depends on the number of hello messages, RREQs, RREPs and RERRs. Due to the incorporation of energy efficient method, routing overhead is comparatively reduced as shown in figure 6 . The consolidated results of the simulation taken for 125 nodes are shown in figure 7 . The results are compared with existing reactive routing protocol (AODV) and proposed methods. 
International Journal of Computer Networks \& Communications (IJCNC) Vol.5, No.2, March 2013

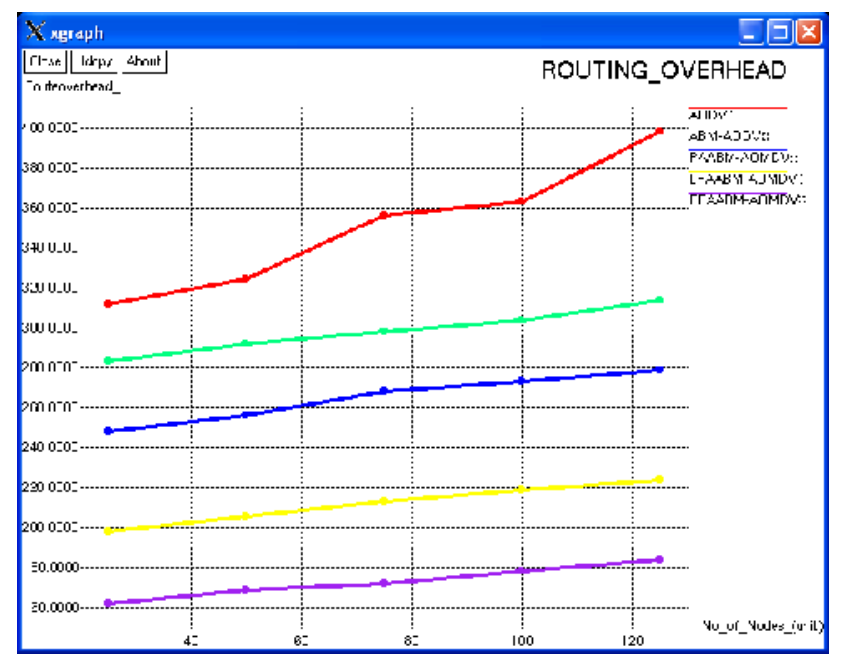

Figure 6. Routing Overhead

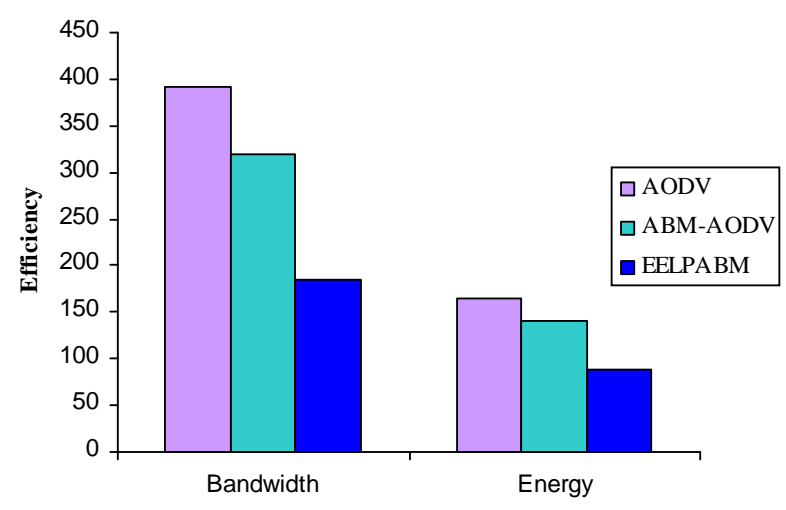

Figure 7. Bandwidth and Energy Consumption

With the above simulation analysis, it can be understood that proposed method improves the performance of ad hoc networks in terms of bandwidth and energy consumption. This method efficiently saves network resources and avoids unexpected loss of QoS guarantees due to collision and malicious attacks.

\section{Conclusion}

The unique characteristics of MANETs make routing a challenging task. Mobility of nodes cause frequent route failure. As a result of these, an effective routing protocol has to adapt to dynamic topology and designed to be bandwidth and energy efficient. Log and pipelined concepts help to reduce the channel idle time and collision overhead. In order to reduce energy consumed by overhearing nodes, probability based method is implemented. Results presented in this article confirmed that the proposed method outperforms the existing method in terms of QoS parameters. Hence, this method improves the available bandwidth and reduces energy consumed by overhearing nodes so that as much as possible bandwidth is available for actual data transmission. 
International Journal of Computer Networks \& Communications (IJCNC) Vol.5, No.2, March 2013

\section{REFERENCES}

[1] L. Chen and W. Heinzelman, "QoS-aware Routing Based on Bandwidth Estimation for Mobile Ad Hoc Networks", IEEE Journal on Selected Areas of Communication, Vol 23, No 3, 2005.

[2] T. Bheemarjuna Reddy, I. Karthigeyan, B.S. Manoj, C. Siva Ram Murthy, "Quality of service provisioning in ad hoc wireless networks: a survey of issues and solutions", Ad Hoc Networks, pp. 83-124(Elsevier), June 2004.

[3] C.E. Perkins and E.M. Royer, "Ad-hoc on-demand distance vector routing", in proceedings of the 2nd IEEE Workshop on Mobile computing Systems and Applications, New Orleans, LA, pp. 90100, Feb 1999.

[4] Perumal Sambasivam, Ashwin Murthy, E. M. Belding-Royer, "Dynamically Adaptive Multipath Routing based on AODV", Proc. 3rd Annual MAHN, 2004.

[5] Cheikh Sarr, Claude Chaudet, Guillaume Chelius, and Isabelle Gue' rin Lassous," Bandwidth Estimation for IEEE 802.11-Based Ad Hoc Networks", IEEE Transactions on Mobile Computing, Vol. 7, No. 10, Oct 2008.

[6] Cheikh Sarr, Claude Chaudet, Guillaume Chelius, Isabelle Guérin Lassous, "Available Bandwidth Estimation for IEEE 802.11-based Ad Hoc networks", INRIA 00154208, Version 2, June 2007.

[7] R. de Renesse, M. Ghassemian, V. Friderikos, A.H Aghvami, "QoS Enabled Routing in Mobile Ad Hoc Networks", IEEE International Conf. on 3G mobile communication Technologies, Oct 2004.

[8] C. Sarr, C. Chaudet, G. Chelius, and I. Guérin Lassous, "A node-based available bandwidth evaluation in IEEE 802.11 ad hoc networks", International Journal of Parallel, Emergent and Distributed Systems, 21(6), 2006.

[9] Mohammed Saghir, Tat-Chee Wan, Rahmat Budiarto, "QoS Multicast Routing Based on Bandwidth Estimation in Mobile Ad Hoc Networks", Proceedings of the Int. Conf. on Computer and Communication Engineering, ICCCE'06 Vol. I, 9-11 May 2006, Kuala Lumpur, Malaysia.

[10] Bharghavan, A. Demers, S. Shenker, and L. Zhang, "MACAW: A Media Access Control Protocol for Wireless LANs,” Proc. SIGCOMM '94 Conf. ACM, pp. 212-225, 1994.

[11] Bianchi, "Performance analysis of the IEEE 802.11 distributed coordination function", IEEE Journal on Selected Areas in Communications, Vol. 18. No. 3, March 2000.

[12] Chunyu Hu, H.Kim, J.C. Hou, "An Analysis of the Binary Exponential Backoff Algorithm in Distributed MAC Protocols”, Technical Report No. UIUCDCS-R-2005-2599 (Engr. No. UILUENG2005-1794), July 2005.

[13] N. Song, B. Kwak, J. Song, and L.E. Miller, "Enhancement of IEEE 802.11 distributed coordination function with exponential increase exponential decrease backoff algorithm", Proc. IEEE VTC 2003Spring, 2003.

[14] J. Deng, P.K. Varshney, and Z.J. Haas, "A new backoff algorithm for the IEEE 802.11 distributed coordination function”, Proc. CNDS 2004, Sandiego, CA, Jan. 2004.

[15] P. Chatzimisios, A.C. Boucouvalas, V. Vitsas, A. Vafiadis, A. Oikonomidis, and P. Huang. "A simple and effective backoff scheme for the IEEE 802.11 MAC protocol", in CITSA, Orlando, Florida, USA, July 2005.

[16] S. Manaseer and M. Ould-kauoa, "A New Backoff Algorithm for MAC Protocol in MANETs", 21st Annual UK Performance Engineering Workshop, pp 159-164, 2005.

[17] Xue Yang and N.H. Vaidya, "A Wireless MAC Protocol Using Implicit Pipelining", IEEE Transactions on Mobile Computing, Vol. 5, No. 3, MAR 2006.

[18] Eun-Sun Jung and Nitin H. Vaidya, "An Energy Efficient MAC Protocol for Wireless LANs", IEEE INFOCOM 2002, New York, U.S.A., June 2002.

[19] Eun-Sun Jung and Nitin Vaidya., "A Power Saving MAC Protocol for Wireless Networks", Technical Report, July 2002.

[20] R. Zheng and R. Kravets, "On-Demand Power Management for Ad Hoc Networks", Adhoc Networks (Elsevier), pages 51-68, Nov 2003.

[21] Tanomsak Khacharoen, Anan Phonphoem, "A Power Saving Mechanism in Ad Hoc Network with Quality of Service Support", International Conference on Information and Communication Technologies (ICT 2003), Bangkok, Thailand, April 2003, pp.119-124.

[22] Z. Li and B. Li, "Probabilistic Power Management for Wireless AdHoc Networks," Mobile Networks and Applications, Vol. 10, No. 5, pp. 771-782, 2005.

[23] C. Sengul and R. Kravets, "Conserving Energy with On-Demand Topology Management," Proc. Second IEEE Int'1 Conf. Mobile Ad Hoc and Sensor Systems (MASS ’05), pp. 10-19, 2005. 
International Journal of Computer Networks \& Communications (IJCNC) Vol.5, No.2, March 2013

[24] S. Lim, C. Yu, and C. Das, "Rcast: A Randomized Communication Scheme for Improving Energy Efficiency in Mobile Ad Hoc Networks," Proc. 25th IEEE Int'l Conf. on Distributed Computing Systems (ICDCS '05), pp. 123-132, 2005.

[25] J. Dorsey and D. Siewiorek, "802.11 Power Management Extensions to Monarch ns," Technical Report CMU-CS-04-183, School of Computer Science, Carnegie Mellon Univ., Dec. 2004.

[26] S. Lim, Chansu Yu, and Chita R. Das, "RandomCast: An Energy-Efficient Communication Scheme for Mobile Ad Hoc Networks", IEEE Transactions on Mobile Computing, Vol. 8, No. 8, August 2009.

[27] NS manual http://www.isi.edu/nsnam/ns/ns-documentation.html.

\section{Authors Biography}

N.Sumathi is working as an Associate Professor in SNR Sons College, Coimbatore. She has 17 years of teaching experience. She has published 7 articles and presented 7 papers in national and international conferences.

Dr.C.P.Sumathi is working as Head and Associate Professor in SDNB Vaishnav College for Women, Chennai. She has 22 years of teaching experience and 7 years of research experience. Her research interests are Image Processing and Neural Networks. She has 13 international publications to her credit. 